The read-out from individual pixels on planar semi-conductor sensors are grouped into clusters to reconstruct the location where a charged particle passed through the sensor. The resolution given by individual pixel sizes is significantly improved by using the information from the charge sharing between pixels. Such analog cluster creation techniques have been used by the ATLAS experiment for many years to obtain an excellent performance. However, in dense environments, such as those inside high-energy jets, the clusters have an increased probability of merging the charge deposited by multiple particles. A neural network based algorithm has been developed for the ATLAS Pixel Detector, in order to identify clusters due to multiple particles and to estimate their position. The algorithm significantly reduces ambiguities in the assignment of Pixel Detector measurement to tracks and improves the position accuracy with respect to standard techniques by taking into account the 2-dimensional charge distribution.

Keywords: Silicon detectors, Pixel detectors, Tracking, Position resolution

\section{Introduction}

Silicon pixel detectors are used in high energy physics experiments to measure with high resolution the position of passing charged particles and reconstruct their trajectories. The ATLAS Pixel Detector [1,2] consists of a barrel part with three layers, at radii of $50.5 \mathrm{~mm}, 88.5 \mathrm{~mm}$ and $122.5 \mathrm{~mm}$, and of two end caps, with three disks each. Most pixels have a size of $50 \mu \mathrm{m}$ $\times 400 \mu \mathrm{m}$. The detector is immersed in $2 \mathrm{~T}$ magnetic field. More details on the detector and its operation can be found in [3].

\title{
Neural network based cluster creation in the ATLAS Pixel Detector
}

\author{
A. Andreazza ${ }^{a}$, on behalf of the ATLAS Collaboration \\ Milano, Italy
}

Email address: attilio.andreazza@mi.infn.it (A. Andreazza)

Preprint submitted to Nuclear Inst. and Methods in Physics Research, ANovember 2, 2012 
A novel technique based on neural networks has been implemented to improve the position resolution and two particle separation of the detector. It has been used for the reprocessing of 2011 data and it is now the default algorithm in track reconstruction. In this paper, the Section 2 summarises the classical method relying on linear interpolation between adjacent pixels [4], while Section 3 introduces the neural network algorithm and Section 4 describes its performance.

\section{Charge sharing algorithm}

The majority charged particles crossing a Pixel Detector module generate signals in more than one pixel. Pixels with deposited charge above a predefined threshold are grouped into clusters if they have a common edge or a common corner. The particle position resolution can be improved by using the amount of charge collected in each pixel, measured from the time-overthreshold (ToT).

The standard reconstruction uses the charge sharing algorithm. It improves the geometrical centre of a cluster, whose coordinates $\left(x_{c}, y_{c}\right)^{1}$ are defined respectively as the midpoint of the first and last rows and columns of the cluster, by adding a correction term dependent on the charge sharing variables, $\Omega_{x}$ and $\Omega_{y}$, which are defined as the ratio between the charge collected respectively in the last row (column) of the cluster and the sum of charges collected in both the first and last rows (columns):

$$
\Omega_{x}=\frac{Q_{\text {last row }}}{Q_{\text {first row }}+Q_{\text {last row }}}, \Omega_{y}=\frac{Q_{\text {last col }}}{Q_{\text {first col }}+Q_{\text {last col }}}
$$

The weights $\Delta_{x}$ and $\Delta_{y}$ for the correction

$$
\left(x_{c}, y_{c}\right) \rightarrow\left(x_{c}+\Delta_{x} \cdot\left(\Omega_{x}-1 / 2\right), y_{c}+\Delta_{y} \cdot\left(\Omega_{y}-1 / 2\right)\right)
$$

are calibrated from data, using the linear dependence of the distance between the extrapolated track and the cluster center on the $\Omega_{x(y)}$ variables. They are computed for different cluster sizes and ranges of the incident angle [5]. Figure 1 shows an example of calibration and resolution improvement with this algorithm. Test beam data demonstrate that this charge sharing algorithm provides resolution down to $\approx 5 \mu \mathrm{m}$ along $x$ for single particles $[2,4]$.

\footnotetext{
${ }^{1}$ The coordinate $x$ (or $r-\phi$ ) refers to the measurement along the $50 \mu \mathrm{m}$ pitch direction, oriented perpendicularly to the magnetic field. The $y$ coordinate runs along the long pitch direction.
} 

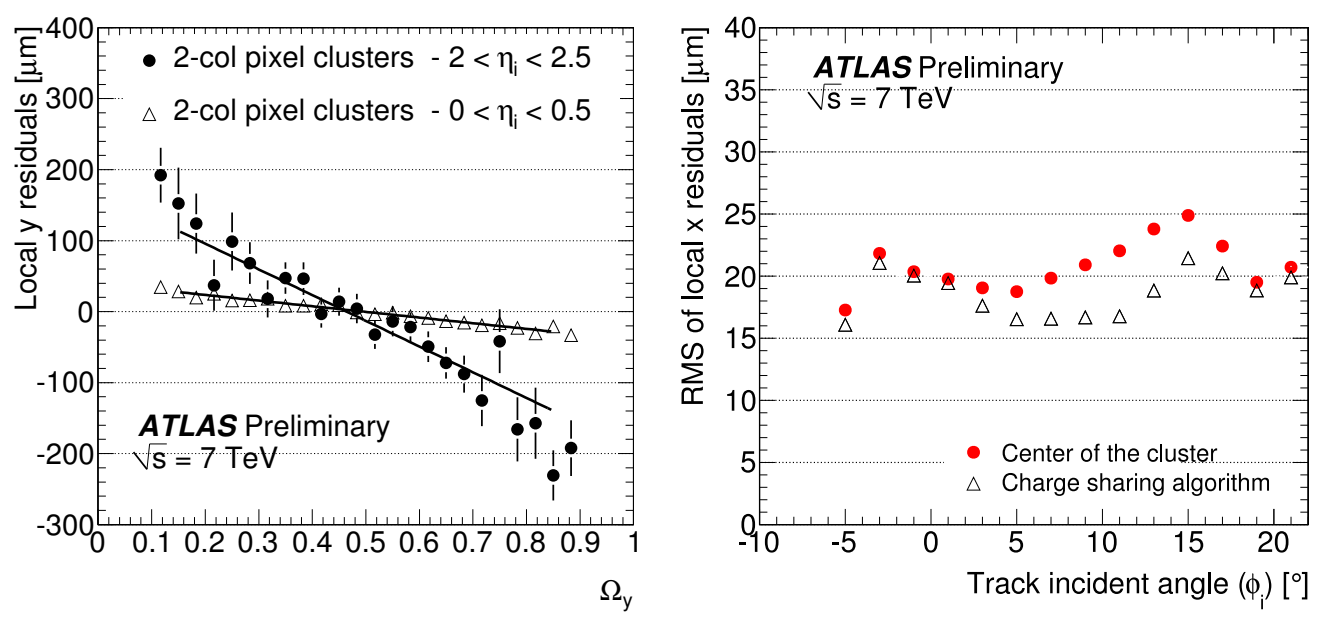

Figure 1: Example of calibration of the linear interpolation constants for the charge sharing algorithm (left) and measured RMS of residuals on the Pixel Detector as a function of the track incidence angle (right). Notice that the RMS includes the contribution from the extrapolation uncertainty, that usually is larger than the detector intrinsic resolution [6].

\section{Neural network algorithm}

In a densely populated track environment (e.g. a jet) two or more particles might deposit charge in adjacent pixels whereby shared clusters are produced. Also $\delta$-ray production may produce large clusters for which a simple linear interpolation is not providing an accurate position reconstruction. The aim of the neural network (NN) algorithm is to improve the two-particle separation and to determine more precise cluster positions by using the detailed information of the cluster shape.

A NN has the ability to solve pattern recognition problems and handle non-linear correlations between input variables. Furthermore, these inputs are differently weighted in the hidden layers of the NN to finally determine the output. These properties of the NN meet the requirements of the cluster reconstruction which has to deal with many cluster properties which have insufficient standalone information (e.g. the charge of a single pixels). When these cluster properties are combined additional information are added (e.g. the knowledge of the charges of adjacent pixels), these variables contain all information required for a successful pattern recognition.

A set of NNs is used to perform all the required tasks, in sequence: 
1. To estimate the number of charged particles passing through a cluster.

2. To estimate the position of the crossing points during the pattern recognition process.

3. To provide a more refined position during the track fitting process, when a preliminary knowledge of track parameters is available.

4. To provide the measurement uncertainty to be used in the track fit.

They consist of feed-forward multilayer perceptrons implemented in the JETNET package [7]. Multiple output nodes are used to determine simultaneously both coordinates of all particles crossing points. For all the NNs the inputs are:

- A matrix of $7 \times 7$ pixels containing the collected charges within the original cluster. The cluster is centered in the matrix by using the charge weights determined by the measured ToT.

- Longitudinal size of the pixels, distinguishing between standard pixels (400 $\mu \mathrm{m}$ long) and longer pixels $(600 \mu \mathrm{m})$.

- Estimated direction of the traversing charged particle.

To train the NN, mixed samples of simulated $t \bar{t}$ and high $p_{\mathrm{T}}$ di-jet events $\left(140<p_{\text {T,jet }}<560 \mathrm{GeV}\right)$ are used. They are generated with MC@NLO [8] and PYTHIA [9], respectively, and processed through a detailed detector simulation [10] based on GEANT4 [11].

\section{Neural network performance}

The non-linear interpolation performed by the NN algorithm is expected to improve the position resolution with respect to the linear interpolation of charge sharing. This is illustrated in Fig. 2, where the difference between tracks extrapolated to the silicon surface and the estimated crossing point positions are shown for clusters of 3- and 4-pixel size along the precision coordinate. Most of these clusters of are due to $\delta$-rays. In such a situation the particle crossing point is usually at one edge of the cluster. The NN algorithm recovers the wide tails of the 3-pixel clusters and the double-peak structure of 4-pixel cluster, which are found using the charge sharing algorithm. Similar improvements can be also observed for other clusters sizes or for the residual distribution in $y$. 

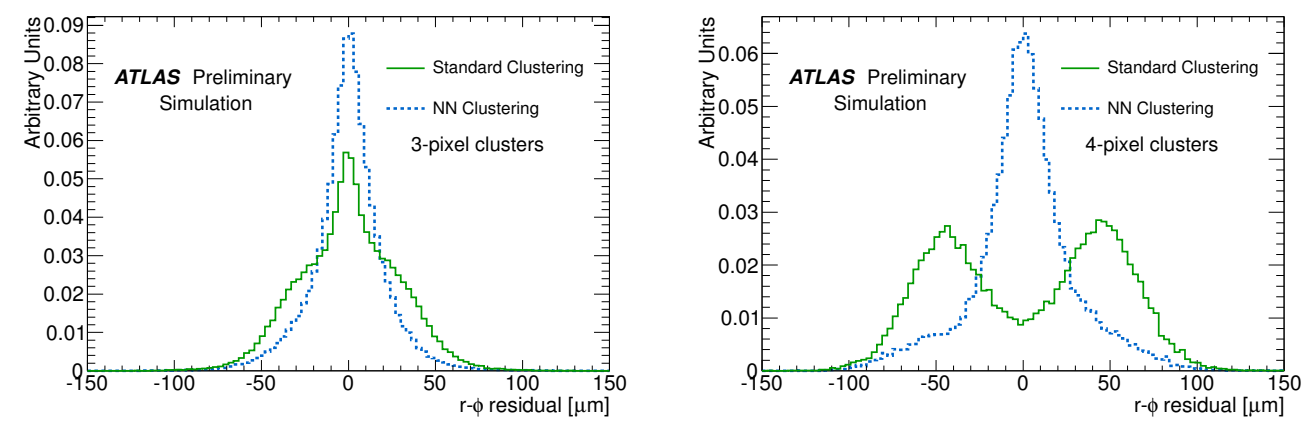

Figure 2: Residuals between the extrapolated track and the measurement on the silicon surface in the transverse plane, for clusters of 3-pixel width (left) and 4-pixel width (right). The continuous line corresponds to the charge sharing algorithm, the dashed line to the neural network. Clusters of these sizes are mostly due to delta rays and overlapping particles, which are better reconstructed by the neural-network based method [12].

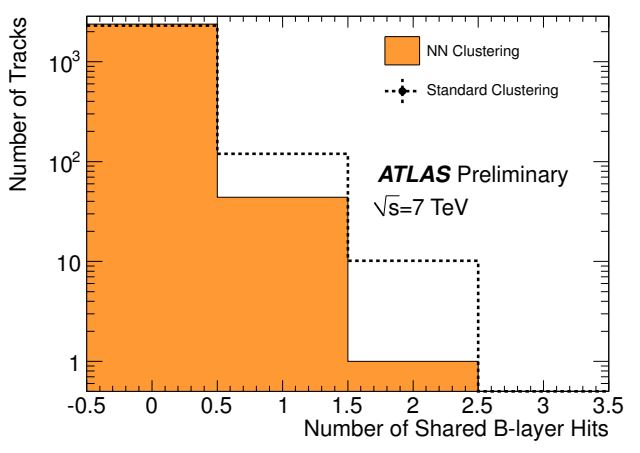

Figure 3: Number of shared hits per tracks with $p_{\mathrm{T}}>100 \mathrm{GeV}$ in the innermost pixel layer. The line corresponds to the charge sharing algorithm, the filled histogram to the neural network [12]. 

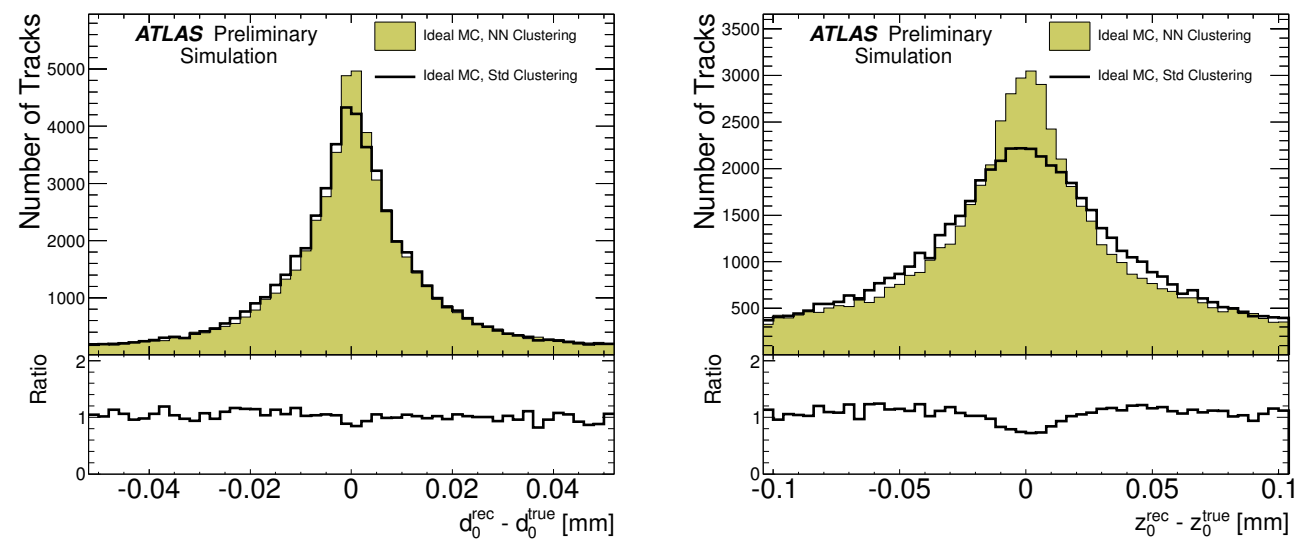

Figure 4: Impact parameter distribution in the transverse plane (left) and along the beam direction (right) for tracks with $p_{\mathrm{T}}>10 \mathrm{GeV}$ and at least one measurement on the innermost pixel layer. The line corresponds to the charge sharing algorithm, the filled histogram to the neural network method [12].

As shared clusters are produced in densely populated track environments, some clusters are associated to multiple tracks. This is particularly relevant for the innermost pixel layer which is as close as $50.5 \mathrm{~mm}$ to the interaction point. The number of these shared hits on-track is shown in Fig. 3 for tracks with $p_{\mathrm{T}}>100 \mathrm{GeV}$, where detector resolution is critical. They are strongly reduced when using the $\mathrm{NN}$ algorithm, because the $\mathrm{NN}$ is able to distinguish the multiple crossing points and provides precise measurements on each track.

Both the more refined interpolation and the reduction of ambiguities provide an improvement in the cluster position resolution. This has direct consequences on the impact parameter measurement, which is the main component of secondary vertex reconstruction and $b$-tagging algorithms. This is shown in Fig. 4. In particular, the longitudinal impact parameter is improved by approximately $15 \%$ for moderate momentum tracks for which the improvements by the NN reconstruction are already striking. The transverse impact parameter shows a similar improvement with the NN algorithm.

\section{Conclusion}

The NN algorithm significantly improves tracking performance of the ATLAS experiment. The improvements coming from the NN reconstruction will 
appear even more relevant when reaching the design luminosity of the LHC and in future upgrades, because of the higher particle density and the greater importance on high- $p_{\mathrm{T}}$ jets. During the 2013 shutdown, the ATLAS Pixel Detector will be upgraded with the insertion of a new layer closer to the interaction point $(R=33.25 \mathrm{~mm})$ [13]. In this extremely dense environment the improved two particle separation power of the NN algorithm will extend the physics potentiality of the ATLAS experiment.

\section{References}

[1] ATLAS Collaboration, The ATLAS Experiment at the CERN Large Hadron Collider, JINST 3 (2008) S08003.

[2] G. Aad et al., ATLAS pixel detector electronics and sensors, JINST 3 (2008) P07007.

[3] A. Andreazza, Status of the ATLAS Pixel Detector at the LHC and its performance after three years of operations, these proceedings, 2012.

[4] I. Gorelov et al., A measurement of Lorentz angle and spatial resolution of radiation hard silicon pixel sensors, Nuclear Instr. and Methods A 481 (2002) 204-221.

[5] S. Montesano, Commissioning of the tracking system of the ATLAS detector, Ph.D. thesis, Università degli Studi di Milano, CERN-THESIS2010-018, https://cdsweb.cern.ch/record/1235187, 2010.

[6] ATLAS Collaboration, https://twiki.cern.ch/twiki/bin/view/AtlasPublic/PixelPublicResults, 2012.

[7] C. Peterson, T. Rögnvaldsson, L. Lönnblad, JETNET 3.0-A versatile artificial neural network package, Comput. Phys. Commun. 81 (1994) $185-220$.

[8] S. Frixione, B. R. Webber, Matching NLO QCD computations and parton shower simulations, JHEP 06 (2002) 029.

[9] T. Sjoestrand, S. Mrenna and P. Skands, PYTHIA 6.4 physics and manual, JHEP 05 (2006) 026. 
141

[10] ATLAS Collaboration, The ATLAS Simulation Infrastructure, Eur. Phys. J. C 70 (2010) 723-753.

[11] S. Agostinelli, et al., GEANT4: A simulation toolkit, Nucl. Instr. and Meth. A 506 (2003) 250-303.

[12] ATLAS Collaboration, http://atlas.web.cern.ch/Atlas/GROUPS/PHYSICS/IDTRACKING/PublicPlots/ATL-COM-PHYS-2012-476/, 2012.

[13] ATLAS Collaboration, ATLAS Insertable B-Layer Technical Design Report, 2010. CERN-LHCC-2010-013. 